\title{
Zero density estimates of $L$-functions associated with cusp forms
}

\author{
by
}

\author{
YuiChi KamiYA (Nagoya)
}

1. Introduction. Let $k$ be a positive even integer, and $f(z)=$ $\sum_{n=1}^{\infty} a(n) e^{2 \pi i n z}$ a holomorphic cusp form of weight $k$ with respect to $\Gamma=S L_{2}(Z)$. We denote by $S_{k}(\Gamma)$ the space of those functions. Let $q$ be a positive integer, and $\chi$ a Dirichlet character $\bmod q$. Let $s=\sigma+i t$ be a complex variable. We define the $L$-function by

$$
L_{f}(s, \chi)=\sum_{n=1}^{\infty} \frac{\chi(n) a(n)}{n^{s}}
$$

for $\sigma>(k+1) / 2$. Denote by $\chi^{*}$ the primitive character mod $q_{1}$ inducing $\chi$. It is known that the function $L_{f}\left(s, \chi^{*}\right)$ has an analytic continuation to the whole complex plane and satisfies the functional equation (see [5])

$$
\left(\frac{2 \pi}{q_{1}}\right)^{-s} \Gamma(s) L_{f}\left(s, \chi^{*}\right)=i^{k}\left(\frac{W\left(\chi^{*}\right)}{\left|W\left(\chi^{*}\right)\right|}\right)^{2}\left(\frac{2 \pi}{q_{1}}\right)^{s-k} \Gamma(k-s) L_{f}\left(k-s, \overline{\chi^{*}}\right),
$$

where $W\left(\chi^{*}\right)$ is Gaussian sum and $\Gamma(s)$ is the gamma function. Moreover, if the cusp form $f$ is the normalized eigenform, that is, the eigenfunction of all Hecke operators with $a(1)=1$, then $a(n)$ 's are real numbers and $L_{f}(s, \chi)$ has the Euler product expansion

$$
L_{f}(s, \chi)=\prod_{p}\left(1-\chi(p) a(p) p^{-s}+\chi(p)^{2} p^{k-1-2 s}\right)^{-1}
$$

for $\sigma>(k+1) / 2$, where the product runs over all prime numbers. Therefore, $L_{f}(s, \chi)$ has the representation

$$
L_{f}(s, \chi)=L_{f}\left(s, \chi^{*}\right) \prod_{p \mid q}\left(1-\chi^{*}(p) a(p) p^{-s}+\chi^{*}(p)^{2} p^{k-1-2 s}\right),
$$

and (1) gives the analytic continuation of $L_{f}(s, \chi)$ to the whole complex plane for every $\chi$. We can also see that $L_{f}(s, \chi)$ has no zeros for $\sigma>(k+1) / 2$,

1991 Mathematics Subject Classification: 11M26, $11 \mathrm{~N} 75$. 
has simple zeros at non-positive integers, and has no zeros for $\sigma<(k-1) / 2$ except non-positive integers. We call zeros at non-positive integers trivial, and those lying in $(k-1) / 2 \leq \sigma \leq(k+1) / 2$ non-trivial. Since $a(n)$ 's are real, we have the relation $\overline{L_{f}(\bar{s}, \bar{\chi})}=L_{f}(s, \chi)$ for any $s$. If $\chi$ is a primitive character, from this relation and the functional equation, non-trivial zeros of $L_{f}(s, \chi)$ are distributed symmetrically with respect to the line $\sigma=k / 2$. In case $\chi$ is an imprimitive character, non-trivial zeros of $L_{f}(s, \chi)$ are those of $L_{f}\left(s, \chi^{*}\right)$ and infinite zeros on $\sigma=(k-1) / 2$ which are coming from the finite products in (1).

For the purpose of counting the number of non-trivial zeros, we define $N_{f}(T, \chi)$
$\quad=\sharp\left\{\varrho=\beta+i \gamma \mid L_{f}(\varrho, \chi)=0,(k-1) / 2 \leq \beta \leq(k+1) / 2,-T \leq \gamma \leq T\right\}$,
$N_{f}\left(\sigma_{0}, T, \chi\right)=\sharp\left\{\varrho=\beta+i \gamma \mid L_{f}(\varrho, \chi)=0, \sigma_{0} \leq \beta \leq(k+1) / 2,-T \leq \gamma \leq T\right\}$

for $\sigma_{0} \geq k / 2$. We can show the following results by modifying the proof for the case of Dirichlet $L$-functions in an obvious way (see [1]). We have

$$
N_{f}(T+1, \chi)-N_{f}(T-1, \chi) \leq C \log (q(T+2))
$$

for any $T \geq 1$ and some positive constant $C$. We also have

$$
N_{f}(T, \chi)=\frac{2 T}{\pi} \log \frac{T}{2 \pi}+O(T \log (q+1)), \quad T \rightarrow \infty,
$$

uniformly in $q$. In particular, for a primitive character $\chi$,

$$
N_{f}(T, \chi)=\frac{2 T}{\pi} \log \frac{q T}{2 \pi}-\frac{2 T}{\pi}+O(\log (q T)), \quad T \rightarrow \infty,
$$

uniformly in $q$.

The purpose of this paper is to show the following theorem.

TheOREM 1. Let $f \in S_{k}(\Gamma)$ be the normalized eigenform and $\chi a$ Dirichlet character mod $q$. If $q \ll T$, then

$$
\sum_{\chi} N_{f}\left(\sigma_{0}, T, \chi\right) \ll(q T)^{\frac{k+1-2 \sigma_{0}}{k / 2+1-\sigma_{0}}}(\log (q T))^{69}, \quad T \rightarrow \infty,
$$

uniformly in $\sigma_{0}$ and $q$ for $k / 2+1 / \log (q T) \leq \sigma_{0} \leq k / 2+1 / 3$, and

$$
\sum_{\chi} N_{f}\left(\sigma_{0}, T, \chi\right) \ll(q T)^{3\left(k+1-2 \sigma_{0}\right) / 2}(\log (q T))^{100}, \quad T \rightarrow \infty,
$$

uniformly in $\sigma_{0}$ and $q$ for $k / 2+1 / 3 \leq \sigma_{0} \leq(k+1) / 2$, where $\sum_{\chi}$ means a sum running over all Dirichlet characters mod $q$.

Specialising $q=1$ in Theorem 1, we have

$$
N_{f}\left(\sigma_{0}, T, \chi_{0}\right) \ll T^{\frac{k+1-2 \sigma_{0}}{k / 2+1-\sigma_{0}}}(\log T)^{69}, \quad T \rightarrow \infty,
$$


uniformly for $k / 2+1 / \log T \leq \sigma_{0} \leq k / 2+1 / 3$,

$$
N_{f}\left(\sigma_{0}, T, \chi_{0}\right) \ll T^{3\left(k+1-2 \sigma_{0}\right) / 2}(\log T)^{100}, \quad T \rightarrow \infty,
$$

uniformly for $k / 2+1 / 3 \leq \sigma_{0} \leq(k+1) / 2$, where $\chi_{0}$ is the trivial character. As regards the estimate of $N_{f}\left(\sigma_{0}, T, \chi_{0}\right)$, Ivić has shown in [4] that

$$
N_{f}\left(\sigma_{0}, T, \chi_{0}\right) \ll T^{\frac{k+1-2 \sigma_{0}}{k / 2+1-\sigma_{0}}+\varepsilon}, \quad T \rightarrow \infty,
$$

for $k / 2 \leq \sigma_{0} \leq k / 2+1 / 4$,

$$
N_{f}\left(\sigma_{0}, T, \chi_{0}\right) \ll T^{\frac{k+1-2 \sigma_{0}}{\sigma_{0}-(k-1) / 2}+\varepsilon}, \quad T \rightarrow \infty,
$$

for $k / 2+1 / 4 \leq \sigma_{0} \leq(k+1) / 2$, and also has shown sharper bounds when $\sigma_{0}$ is near $(k+1) / 2$. Therefore, Theorem 1 is a natural extension of Ivić's results for $k / 2+1 / \log T \leq \sigma_{0} \leq k / 2+1 / 4$.

Theorem 1 is an analogue of zero density estimates of Dirichlet $L$-functions by Montgomery [6]. Montgomery used the estimate of the mean fourth power of Dirichlet $L$-functions on the critical line for this problem. Since the corresponding fourth power result is not known in our case, we shall use the mean square of $L_{f}(s, \chi)$ to prove Theorem 1 (see Theorem 2 in Section 3). To estimate the mean square of $L_{f}(s, \chi)$, we reduce the problem to the study of the mean square of the Dirichlet polynomial by using the approximate functional equation of $L_{f}(s, \chi)$, which is proved by applying the method of Good [3].

The author expresses his sincere gratitude to Professor Kohji Matsumoto and Professor Yoshio Tanigawa for their advice and encouragement.

2. The approximate functional equation. Throughout this section, we suppose $f$ is in $S_{k}(\Gamma)$ and $\chi$ is a primitive character $\bmod q$. We shall prove the approximate functional equation of $L_{f}(s, \chi)$ whose implied constant is uniform in $q$, following the method of Good [3].

Rankin has shown in [7] that

$$
\sum_{n \leq x}|a(n)|^{2}=C x^{k}+O\left(x^{k-2 / 5}\right), \quad x \rightarrow \infty,
$$

where $C$ is a positive constant depending on $k$. By Cauchy's inequality,

$$
\sum_{n \leq x}|a(n)| \ll x^{(k+1) / 2}, \quad x \rightarrow \infty
$$

hence we obtain the following lemma by partial summation.

Lemma 1. Let $\sigma$ be a real number. Then

$$
\sum_{n \leq x}|a(n)| n^{-\sigma} \ll x^{(k+1) / 2-\sigma}, \quad x \rightarrow \infty,
$$


uniformly for $\sigma \leq \sigma_{1}<(k+1) / 2$, and

$$
\begin{aligned}
\sum_{n \leq x}|a(n)|^{2} n^{-2 \sigma} & \\
& \ll \begin{cases}x^{k-2 \sigma} & \text { uniformly for } \sigma \leq \sigma_{2}<k / 2, \\
\log x & \text { uniformly for } k / 2-1 / \log x \leq \sigma \leq k / 2+1 / \log x,\end{cases}
\end{aligned}
$$

where $\sigma_{1}$ and $\sigma_{2}$ are constants.

Following the notation in [3], let $\varphi(\varrho)$ be a real-valued function in $[0, \infty)$ which is infinitely differentiable and satisfies $\varphi(\varrho)=1$ for $0 \leq \varrho \leq 1 / 2$ and $\varphi(\varrho)=0$ for $\varrho \geq 2$. We denote by $\Phi$ the set of those functions. The function $\varphi_{0}(\varrho)=1-\varphi(1 / \varrho)$ is also an element of $\Phi$. For $\varphi$ in $\Phi$ and for a complex variable $w=u+i v$ with $u>0$, let

$$
K_{\varphi}(w)=w \int_{0}^{\infty} \varphi(\varrho) \varrho^{w-1} d \varrho .
$$

The function $K_{\varphi}(w)$ has an analytic continuation to the whole complex $w$-plane, because the relation

$$
K_{\varphi}(w)=-\int_{1 / 2}^{2} \varphi^{\prime}(\varrho) \varrho^{w} d \varrho
$$

can be verified by integration by parts. Let $\varphi^{(j)}$ denote the $j$ th derivative of $\varphi$ and define

$$
\left\|\varphi^{(j)}\right\|_{1}=\int_{0}^{\infty}\left|\varphi^{(j)}(\varrho)\right| d \varrho .
$$

For $\tau>0, t \neq 0$, and $j=0,1, \ldots$, let

$$
\gamma_{j}(s, \tau)=\frac{1}{2 \pi i \Gamma(s)} \int_{\mathcal{F}} \Gamma(s+w) \frac{\left(\tau \exp \left(-i \frac{\pi}{2} \operatorname{sgn}(t)\right)\right)^{w}}{w(w+1) \ldots(w+j)} d w,
$$

where $\operatorname{sgn}(t)=t /|t|$ and $\int_{\mathcal{F}}$ means that integration is taken over the curve $\mathcal{F}$ which encircles $w=0,-1, \ldots,-j$. If $j=0$, it is easy to see that $\gamma_{0}(s, \tau)=1$ for any $s$. In case $j \neq 0$, it was shown in [3] that

$$
\gamma_{j}\left(s,|t|^{-1}\right) \ll \begin{cases}|t|^{-(j+1) / 2} & \text { for odd } j, \\ |t|^{-j / 2} & \text { for even } j,\end{cases}
$$

uniformly for $\sigma$ which is in a fixed strip. For $x>0$ and $\varphi$ in $\Phi$, let

$$
\begin{aligned}
G_{f}(s, x ; \varphi, \chi)= & \frac{1}{2 \pi i \Gamma(s)} \int_{(k / 2+1-\sigma)} \Gamma(s+w) L_{f}(s+w, \chi) \frac{K_{\varphi}(w)}{w} \\
& \times\left(\frac{q x}{2 \pi} \exp \left(-i \frac{\pi}{2} \operatorname{sgn}(t)\right)\right)^{w} d w
\end{aligned}
$$


where $\int_{(k / 2+1-\sigma)}$ means that integration is taken over the vertical line $u=$ $k / 2+1-\sigma$.

We can derive the following lemma by modifying Satz of [3].

Lemma 2. Let $x>0, \varphi \in \Phi, f \in S_{k}(\Gamma)$, and $\chi$ a primitive character mod $q$. Then the following properties hold.

(a) For $(k-1) / 2 \leq \sigma \leq(k+1) / 2$,

$$
\begin{aligned}
\left(\frac{2 \pi}{q}\right)^{-s} \Gamma(s) L_{f}(s, \chi)= & \left(\frac{2 \pi}{q}\right)^{-s} \Gamma(s) G_{f}(s, x ; \varphi, \chi) \\
& +i^{k}\left(\frac{W(\chi)}{|W(\chi)|}\right)^{2}\left(\frac{2 \pi}{q}\right)^{s-k} \Gamma(k-s) \\
& \times G_{f}\left(k-s, x^{-1} ; \varphi_{0}, \bar{\chi}\right) .
\end{aligned}
$$

(b) Let $y=q x|t| /(2 \pi)$ and $l$ an integer with $l>(k+1) / 2$. For $|t|>l^{2}$,

$$
\begin{aligned}
G_{f}(s, x ; \varphi, \chi)= & \sum_{n=1}^{\infty} \frac{\chi(n) a(n)}{n^{s}} \sum_{j=0}^{l} \varphi^{(j)}\left(\frac{n}{y}\right)\left(-\frac{n}{y}\right)^{j} \gamma_{j}\left(s,|t|^{-1}\right) \\
& +O\left(\left\|\varphi^{(l+1)}\right\|_{1} y^{(k+1) / 2-\sigma}|t|^{-l / 2}\right),
\end{aligned}
$$

where the implied constant is uniform in $\sigma, \varphi$, and $q$ for $(k-1) / 2 \leq \sigma \leq$ $(k+1) / 2$.

Put $x=1$ and $y=q|t| /(2 \pi)$ in Lemma 2. Then we have

$$
\begin{aligned}
L_{f}(s, \chi)= & \sum_{n=1}^{\infty} \frac{\chi(n) a(n)}{n^{s}} \varphi\left(\frac{n}{y}\right) \\
& +i^{k}\left(\frac{W(\chi)}{|W(\chi)|}\right)^{2}\left(\frac{2 \pi}{q}\right)^{2 s-k} \frac{\Gamma(k-s)}{\Gamma(s)} \sum_{n=1}^{\infty} \frac{\bar{\chi}(n) a(n)}{n^{k-s}} \varphi_{0}\left(\frac{n}{y}\right) \\
& +R(s),
\end{aligned}
$$

where

$$
\begin{aligned}
R(s)= & \sum_{n=1}^{\infty} \frac{\chi(n) a(n)}{n^{s}} \sum_{j=1}^{l} \varphi^{(j)}\left(\frac{n}{y}\right)\left(-\frac{n}{y}\right)^{j} \gamma_{j}\left(s,|t|^{-1}\right) \\
& +i^{k}\left(\frac{W(\chi)}{|W(\chi)|}\right)^{2}\left(\frac{2 \pi}{q}\right)^{2 s-k} \frac{\Gamma(k-s)}{\Gamma(s)} \\
& \times \sum_{n=1}^{\infty} \frac{\bar{\chi}(n) a(n)}{n^{k-s}} \sum_{j=1}^{l} \varphi_{0}^{(j)}\left(\frac{n}{y}\right)\left(-\frac{n}{y}\right)^{j} \gamma_{j}\left(k-s,|t|^{-1}\right) \\
& +O\left(\left\|\varphi^{(l+1)}\right\|_{1} y^{(k+1) / 2-\sigma}|t|^{-l / 2}\right)+O\left(\left\|\varphi_{0}^{(l+1)}\right\|_{1} y^{(k+1) / 2-\sigma}|t|^{-l / 2}\right) .
\end{aligned}
$$


Now we fix a $\varphi$. By (5) and (7), we have

$$
\begin{aligned}
R(s) \ll & \sum_{j=1}^{l}\left|\gamma_{j}\left(s,|t|^{-1}\right)\right| \sum_{n \leq 2 y} \frac{|a(n)|}{n^{\sigma}}\left(\frac{n}{q|t|}\right)^{j} \\
& +\left|\left(\frac{2 \pi}{q}\right)^{2 s-k} \frac{\Gamma(k-s)}{\Gamma(s)}\right| \sum_{j=1}^{l}\left|\gamma_{j}\left(k-s,|t|^{-1}\right)\right| \sum_{n \leq 2 y} \frac{|a(n)|}{n^{k-\sigma}}\left(\frac{n}{q|t|}\right)^{j} \\
& +(q|t|)^{(k+1) / 2-\sigma}|t|^{-l / 2} \\
\ll & (q|t|)^{(k+1) / 2-\sigma}|t|^{-1} .
\end{aligned}
$$

Therefore we have

Lemma 3. Let $\varphi \in \Phi, f \in S_{k}(\Gamma), \chi$ a primitive character mod $q$, and $\kappa=2 \pi / q$. Then

$$
\begin{aligned}
L_{f}(s, \chi)= & \sum_{n=1}^{\infty} \frac{\chi(n) a(n)}{n^{s}} \varphi\left(\frac{\kappa n}{|t|}\right) \\
& +i^{k}\left(\frac{W(\chi)}{|W(\chi)|}\right)^{2} \kappa^{2 s-k} \frac{\Gamma(k-s)}{\Gamma(s)} \sum_{n=1}^{\infty} \frac{\bar{\chi}(n) a(n)}{n^{k-s}} \varphi_{0}\left(\frac{\kappa n}{|t|}\right) \\
& +O\left((q|t|)^{(k+1) / 2-\sigma}|t|^{-1}\right),
\end{aligned}
$$

where the implied constant is uniform in $\sigma$ and $q$ for $(k-1) / 2 \leq \sigma \leq$ $(k+1) / 2$.

3. The mean square of $L_{f}(s, \chi)$. Throughout this section, we suppose $f$ is in $S_{k}(\Gamma)$ and $\chi$ is a Dirichlet character $\bmod q$. The aim of this section is to estimate the mean square

$$
\sum_{\chi}^{*} \int_{-T}^{T}\left|L_{f}(\sigma+i t, \chi)\right|^{2} d t
$$

uniformly in $\sigma$ and $q$ for $k / 2-1 / \log (q T) \leq \sigma \leq k / 2+1 / \log (q T)$, where $\sum_{\chi}^{*}$ means a sum running over all primitive characters $\bmod q$.

We need the following lemmas.

Lemma 4. Let $0<\delta<\delta_{1}$, and let $\varphi(\varrho)$ be a real-valued function in $[0, \infty)$ which is twice continuously differentiable and satisfies $\varphi(\varrho)=1$ for $0 \leq \varrho \leq \delta$ and $\varphi(\varrho)=0$ for $\varrho \geq \delta_{1}$. Let $m$ and $n$ be positive integers, $\kappa$ and $T$ positive real numbers, and $\beta$ a real number which satisfies $-1<A \leq \beta \leq B<1$ for some constants $A, B$. Then 


$$
\begin{aligned}
& \int_{0}^{T} \varphi\left(\frac{\kappa n}{t}\right) \varphi\left(\frac{\kappa m}{t}\right) t^{-\beta} \cos \left(t \log \frac{n}{m}\right) d t \\
& = \begin{cases}0 & \text { for } m \geq T \delta_{1} / \kappa \text { or } n \geq T \delta_{1} / \kappa, \\
O\left(T^{1-\beta}\right) & \text { for } m=n<T \delta / \kappa, \\
O\left((\kappa n)^{1-\beta}\right) & \text { for } m=n \geq T \delta / \kappa, \\
\frac{1}{\log \frac{n}{m}} \sin \left(T \log \frac{n}{m}\right) \varphi\left(\frac{\kappa n}{T}\right) \varphi\left(\frac{\kappa m}{T}\right) T^{-\beta}+O\left(\frac{(\kappa \max (n, m))^{-\beta-1}}{(\log (n / m))^{2}}\right) \\
\text { for } m \neq n,\end{cases}
\end{aligned}
$$

where the implied constants are uniform in $m, n, \kappa$, and $\beta$.

It is easy to prove Lemma 4 by modifying the proof of Lemma 7 of [3].

Lemma 5. Let $f \in S_{k}(\Gamma)$ and $\chi$ a Dirichlet character mod $q$. Let $\varepsilon$ be a positive real number and assume $(k-\varepsilon) / 2<\sigma<(k+\varepsilon) / 2$. If $|t| \leq C$ for some positive constant $C$, then

$$
\sum_{\chi}^{*}\left|L_{f}(s, \chi)\right|^{2} \ll_{\varepsilon, C} \phi(q) q^{k-2 \sigma+2 \varepsilon}\left(\int_{1}^{\infty} u^{2 \sigma-k-1-\varepsilon} d u+\int_{1}^{\infty} u^{k-2 \sigma-1-\varepsilon} d u\right)
$$

uniformly in $\sigma$ and $q$, where $\phi$ is the Euler function.

Proof. By the automorphic property of $\sum_{n=1}^{\infty} \chi(n) a(n) e^{2 \pi i n z}$, which is the twist of $f$ by the primitive character $\chi$,

$$
\begin{aligned}
\left(\frac{2 \pi}{q}\right)^{-s} \Gamma(s) L_{f} & (s, \chi) \\
= & \int_{0}^{\infty} u^{s-1} \sum_{n=1}^{\infty} \chi(n) a(n) e^{-2 \pi n u / q} d u \\
= & \int_{1}^{\infty} u^{s-1} \sum_{n=1}^{\infty} \chi(n) a(n) e^{-2 \pi n u / q} d u \\
& +i^{k}\left(\frac{W(\chi)}{|W(\chi)|}\right)^{2} \int_{1}^{\infty} u^{k-s-1} \sum_{n=1}^{\infty} \bar{\chi}(n) a(n) e^{-2 \pi n u / q} d u
\end{aligned}
$$

Hence

$$
\begin{aligned}
\left(\frac{2 \pi}{q}\right)^{-\sigma}|\Gamma(s)| \cdot\left|L_{f}(s, \chi)\right| \leq & \int_{1}^{\infty} u^{\sigma-1}\left|\sum_{n=1}^{\infty} \chi(n) a(n) e^{-2 \pi n u / q}\right| d u \\
& +\int_{1}^{\infty} u^{k-\sigma-1}\left|\sum_{n=1}^{\infty} \bar{\chi}(n) a(n) e^{-2 \pi n u / q}\right| d u .
\end{aligned}
$$

By squaring both sides above and taking $\sum_{\chi}^{*}$, we have 
(8) $\frac{1}{2}\left(\frac{2 \pi}{q}\right)^{-2 \sigma}|\Gamma(s)|^{2} \sum_{\chi}^{*}\left|L_{f}(s, \chi)\right|^{2}$

$$
\begin{aligned}
\leq & \sum_{\chi}^{*}\left(\int_{1}^{\infty} u^{\sigma-1}\left|\sum_{n=1}^{\infty} \chi(n) a(n) e^{-2 \pi n u / q}\right| d u\right)^{2} \\
& +\sum_{\chi}^{*}\left(\int_{1}^{\infty} u^{k-\sigma-1}\left|\sum_{n=1}^{\infty} \bar{\chi}(n) a(n) e^{-2 \pi n u / q}\right| d u\right)^{2} .
\end{aligned}
$$

Let $\alpha$ be real. By Cauchy's inequality,

$$
\begin{aligned}
\sum_{\chi}^{*}\left(\int_{1}^{\infty} u^{\alpha-1} \mid\right. & \left.\sum_{n=1}^{\infty} \chi(n) a(n) e^{-2 \pi n u / q} \mid d u\right)^{2} \\
& \leq \sum_{\chi}^{*} \int_{1}^{\infty} u^{2 \alpha-1+\varepsilon}\left|\sum_{n=1}^{\infty} \chi(n) a(n) e^{-2 \pi n u / q}\right|^{2} d u \int_{1}^{\infty} u^{-1-\varepsilon} d u \\
& \ll \varepsilon \int_{1}^{\infty} u^{2 \alpha-1+\varepsilon} \sum_{\chi}\left|\sum_{n=1}^{\infty} \chi(n) a(n) e^{-2 \pi n u / q}\right|^{2} d u .
\end{aligned}
$$

Here,

$$
\begin{aligned}
\sum_{\chi}\left|\sum_{n=1}^{\infty} \chi(n) a(n) e^{-2 \pi n u / q}\right|^{2} & \\
& =\phi(q) \sum_{\substack{n=1 \\
(n, q)=1 \\
n \equiv m(q)}}^{\infty} \sum_{\substack{m=1 \\
(m, q)=1}}^{\infty} \bar{a}(n) a(m) e^{-2 \pi(n+m) u / q} \\
& \leq \frac{\phi(q)}{2} \sum_{\substack{n=1 \\
n \equiv m(q)}}^{\infty} \sum_{m=1}^{\infty}\left(|a(n)|^{2}+|a(m)|^{2}\right) e^{-2 \pi(n+m) u / q} \\
& \leq \phi(q) \sum_{n=1}^{\infty}|a(n)|^{2} e^{-2 \pi n u / q} \sum_{r=0}^{\infty} e^{-2 \pi r u} \\
& \ll \phi(q) \sum_{n=1}^{\infty}|a(n)|^{2} e^{-2 \pi n u / q} .
\end{aligned}
$$

By using partial summation, the right-hand side is

$$
\begin{aligned}
& \ll \phi(q) \frac{u}{q} \int_{1}^{\infty} x^{k} e^{-2 \pi x u / q} d x \ll_{\varepsilon} \phi(q) \frac{u}{q} \int_{1}^{\infty} x^{k}\left(\frac{x u}{q}\right)^{-k-1-2 \varepsilon} d x \\
& \ll_{\varepsilon} \phi(q)\left(\frac{u}{q}\right)^{-k-2 \varepsilon} .
\end{aligned}
$$


Hence we have

$$
\sum_{\chi}^{*}\left(\int_{1}^{\infty} u^{\alpha-1}\left|\sum_{n=1}^{\infty} \chi(n) a(n) e^{-2 \pi n u / q}\right| d u\right)^{2} \ll_{\varepsilon} \phi(q) q^{k+2 \varepsilon} \int_{1}^{\infty} u^{2 \alpha-k-1-\varepsilon} d u .
$$

Substituting this into (8), we obtain the assertion of Lemma 5.

Theorem 2. Let $f \in S_{k}(\Gamma)$ and $\chi$ a Dirichlet character mod $q$. If $q \ll T$, then

$$
\sum_{\chi}^{*} \int_{-T}^{T}\left|L_{f}(\sigma+i t, \chi)\right|^{2} d t \ll \phi(q) T \log (q T), \quad T \rightarrow \infty,
$$

uniformly in $\sigma$ and $q$ for $k / 2-1 / \log (q T) \leq \sigma \leq k / 2+1 / \log (q T)$.

Proof. Denote the right-hand side of the formula in the statement of Lemma 3 by $f_{1}+f_{2}+f_{3}$, say. Let $C_{0}$ be a positive constant for which

$$
f_{3}(\sigma+i t) \ll(q|t|)^{(k+1) / 2-\sigma}|t|^{-1}
$$

for $|t| \geq C_{0}$. Put

$$
\Lambda_{\mu \nu}\left(\sigma, C_{0}\right)=\int_{[-T, T]-\left[-C_{0}, C_{0}\right]} \overline{f_{\mu}(\sigma+i t)} f_{\nu}(\sigma+i t) d t, \quad \mu, \nu=1,2,3 .
$$

By Cauchy's inequality,

$$
\begin{aligned}
\left|\sum_{\chi}^{*} \Lambda_{\mu \nu}\left(\sigma, C_{0}\right)\right| & \leq\left(\sum_{\chi}^{*} \Lambda_{\mu \mu}\left(\sigma, C_{0}\right)\right)^{1 / 2}\left(\sum_{\chi}^{*} \Lambda_{\nu \nu}\left(\sigma, C_{0}\right)\right)^{1 / 2} \\
& \leq \frac{1}{2} \sum_{\chi}^{*} \Lambda_{\mu \mu}\left(\sigma, C_{0}\right)+\frac{1}{2} \sum_{\chi}^{*} \Lambda_{\nu \nu}\left(\sigma, C_{0}\right) .
\end{aligned}
$$

Hence we have

$$
\begin{aligned}
\sum_{\chi}^{*} \int_{-T}^{T}\left|L_{f}(\sigma+i t, \chi)\right|^{2} d t & \\
= & \left|\sum_{\mu, \nu=1}^{3} \sum_{\chi}^{*} \Lambda_{\mu \nu}\left(\sigma, C_{0}\right)\right|+\sum_{\chi}^{*} \int_{-C_{0}}^{C_{0}}\left|L_{f}(\sigma+i t, \chi)\right|^{2} d t \\
& \ll \sum_{\nu=1}^{3} \sum_{\chi}^{*} \Lambda_{\nu \nu}\left(\sigma, C_{0}\right)+\sum_{\chi}^{*} \int_{-C_{0}}^{C_{0}}\left|L_{f}(\sigma+i t, \chi)\right|^{2} d t .
\end{aligned}
$$

We use Lemma 5 with $\varepsilon=1 / 2$ for $k / 2-1 / \log (q T) \leq \sigma \leq k / 2+1 / \log (q T)$ and $|t| \leq C_{0}$ to obtain

$$
\sum_{\chi}^{*} \int_{-C_{0}}^{C_{0}}\left|L_{f}(\sigma+i t, \chi)\right|^{2} d t \ll_{C_{0}} \phi(q) q
$$


By (9), we have

$$
\begin{aligned}
\sum_{\chi}^{*} \Lambda_{33}\left(\sigma, C_{0}\right) & \ll \phi(q) q^{2 / \log (q T)+1} \int_{C_{0}}^{T} t^{2 / \log (q T)-1} d t \\
& \ll \phi(q) q \log T .
\end{aligned}
$$

Substituting (11) and (12) into (10), gives

(13) $\sum_{\chi}^{*} \int_{-T}^{T}\left|L_{f}(\sigma+i t, \chi)\right|^{2} d t \ll \sum_{\chi}^{*} \Lambda_{11}(\sigma)+\sum_{\chi}^{*} \Lambda_{22}(\sigma)+\phi(q) q \log T$,

where

$$
\Lambda_{\nu \nu}(\sigma)=\int_{-T}^{T}\left|f_{\nu}(\sigma+i t)\right|^{2} d t, \quad \nu=1,2
$$

First, we estimate $\sum_{\chi}^{*} \Lambda_{11}(\sigma)$. We have

$$
\begin{aligned}
\sum_{\chi}^{*} \Lambda_{11}(\sigma) \leq & \sum_{\chi} \int_{-T}^{T}\left|f_{1}(\sigma+i t)\right|^{2} d t \\
= & 2 \phi(q) \sum_{\substack{n<2 T / \kappa \\
(n, q)=1 \\
n \equiv m(q)}} \sum_{\substack{m<2 T / \kappa \\
(m, q)=1}} \frac{\bar{a}(n) a(m)}{(n m)^{\sigma}} \\
& \times \int_{0}^{T} \varphi\left(\frac{\kappa n}{t}\right) \varphi\left(\frac{\kappa m}{t}\right) \cos \left(t \log \frac{n}{m}\right) d t \\
= & 2 \phi(q)\left\{\sum_{\substack{n<T /(2 \kappa) \\
(n, q)=1}} \frac{|a(n)|^{2}}{n^{2 \sigma}} \int_{0}^{T} \varphi\left(\frac{\kappa n}{t}\right)^{2} d t\right. \\
& +\sum_{\substack{T /(2 \kappa) \leq n<2 T / \kappa \\
(n, q)=1}} \frac{|a(n)|^{2}}{n^{2 \sigma}} \int_{0}^{T} \varphi\left(\frac{\kappa n}{t}\right)^{2} d t \\
& \left.+\sum_{0} \frac{\bar{a}(n) a(m)}{(n m)^{\sigma}} \int_{0}^{T} \varphi\left(\frac{\kappa n}{t}\right) \varphi\left(\frac{\kappa m}{t}\right)^{2} \cos \left(t \log \frac{n}{m}\right) d t\right\}
\end{aligned}
$$

where we set

$$
\sum_{0}=\sum_{\substack{n<2 T / \kappa \\(n, q)=1 \\ n \equiv m(q) \\ n \neq m}} \sum_{\substack{m<2 T / \kappa \\(m, q)=1}}
$$


Applying Lemma 4, we have

$$
\begin{aligned}
\sum_{\chi}^{*} \Lambda_{11}(\sigma) \ll & \phi(q)\left\{T \sum_{n<T /(2 \kappa)} \frac{|a(n)|^{2}}{n^{2 \sigma}}+\frac{1}{q} \sum_{n<2 T / \kappa} \frac{|a(n)|^{2}}{n^{2 \sigma-1}}\right. \\
& +\sum_{0} \frac{|a(n) a(m)|}{(n m)^{\sigma}\left|\log \frac{n}{m}\right|} \\
& \left.+q \sum_{0} \frac{|a(n) a(m)|}{(n m)^{\sigma} \max (n, m)\left(\log \frac{n}{m}\right)^{2}}\right\}
\end{aligned}
$$

The third sum on the right-hand side is

$$
\begin{aligned}
& \leq \sum_{n<2 T / \kappa} \frac{|a(n)|^{2}}{n^{2 \sigma}} \sum_{\substack{m<2 T / \kappa \\
m \equiv n(q) \\
m \neq n}} \frac{1}{\left|\log \frac{n}{m}\right|} \\
& =\sum_{n<2 T / \kappa} \frac{|a(n)|^{2}}{n^{2 \sigma}} \sum_{\substack{m<n \\
m \equiv n(q)}} \frac{1}{\left|\log \frac{n}{m}\right|}+\sum_{n<2 T / \kappa} \frac{|a(n)|^{2}}{n^{2 \sigma}} \sum_{\substack{n<m<2 T / \kappa \\
m \equiv n(q)}} \frac{1}{\left|\log \frac{n}{m}\right|} .
\end{aligned}
$$

In the first term we put $m=n-q r$ to get

$$
\sum_{\substack{m<n \\ m \equiv n(q)}} \frac{1}{\left|\log \frac{n}{m}\right|}<\frac{n}{q} \sum_{1 \leq r<2 T /(q \kappa)} \frac{1}{r} \ll \frac{n}{q} \log T,
$$

and in the second term we put $m=n+q r$ to get

$$
\sum_{\substack{n<m<2 T / \kappa \\ m \equiv n(q)}} \frac{1}{\left|\log \frac{n}{m}\right|}<\sum_{1 \leq r<2 T /(q \kappa)} \frac{n+q r}{q r} \ll T+\frac{n}{q} \log T .
$$

Therefore we have

$$
\sum_{0} \frac{|a(n) a(m)|}{(n m)^{\sigma}\left|\log \frac{n}{m}\right|} \ll T \sum_{n<2 T / \kappa} \frac{|a(n)|^{2}}{n^{2 \sigma}}+\frac{\log T}{q} \sum_{n<2 T / \kappa} \frac{|a(n)|^{2}}{n^{2 \sigma-1}} .
$$

Next, the fourth sum on the right-hand side of (14) is

$$
\begin{aligned}
& \leq \sum_{n<2 T / \kappa} \frac{|a(n)|^{2}}{n^{2 \sigma}} \sum_{\substack{m<2 T / \kappa \\
m \equiv n(q) \\
m \neq n}} \frac{1}{\max (n, m)\left(\log \frac{n}{m}\right)^{2}} \\
& =\sum_{n<2 T / \kappa} \frac{|a(n)|^{2}}{n^{2 \sigma+1}} \sum_{\substack{m<n \\
m \equiv n(q)}} \frac{1}{\left(\log \frac{n}{m}\right)^{2}}+\sum_{n<2 T / \kappa} \frac{|a(n)|^{2}}{n^{2 \sigma}} \sum_{\substack{n<m<2 T / \kappa \\
m \equiv n(q)}} \frac{1}{m\left(\log \frac{n}{m}\right)^{2}} .
\end{aligned}
$$


In the first term we put $m=n-q r$ to get

$$
\sum_{\substack{m<n \\ m \equiv n(q)}} \frac{1}{\left(\log \frac{n}{m}\right)^{2}}<\frac{n^{2}}{q^{2}} \sum_{1 \leq r<n / q} \frac{1}{r^{2}} \ll \frac{n^{2}}{q^{2}},
$$

and in the second term we put $m=n+q r$ to get

$$
\sum_{\substack{n<m<2 T / \kappa \\ m \equiv n(q)}} \frac{1}{m\left(\log \frac{n}{m}\right)^{2}}<\sum_{1 \leq r<2 T /(q \kappa)} \frac{1}{n+q r}\left(\frac{n+q r}{q r}\right)^{2} \ll \frac{n}{q^{2}}+\frac{1}{q} \log T .
$$

Therefore we have

$$
\begin{aligned}
& q \sum_{0} \frac{|a(n) a(m)|}{(n m)^{\sigma} \max (n, m)\left(\log \frac{n}{m}\right)^{2}} \\
& \ll(\log T) \sum_{n<2 T / \kappa} \frac{|a(n)|^{2}}{n^{2 \sigma}}+\frac{1}{q} \sum_{n<2 T / \kappa} \frac{|a(n)|^{2}}{n^{2 \sigma-1}} .
\end{aligned}
$$

Substituting (15) and (16) to (14), we obtain

$$
\sum_{\chi}^{*} \Lambda_{11}(\sigma) \ll \phi(q)\left(T \sum_{n<2 T / \kappa} \frac{|a(n)|^{2}}{n^{2 \sigma}}+\frac{\log T}{q} \sum_{n<2 T / \kappa} \frac{|a(n)|^{2}}{n^{2 \sigma-1}}\right) .
$$

Combining this estimate with (6), we obtain

$$
\sum_{\chi}^{*} \Lambda_{11}(\sigma) \ll \phi(q) T \log (q T), \quad T \rightarrow \infty,
$$

uniformly in $\sigma$ and $q$ for $k / 2-1 / \log (q T) \leq \sigma \leq k / 2+1 / \log (q T)$.

Second, we estimate $\sum_{\chi}^{*} \Lambda_{22}(\sigma)$. We have

$$
\begin{aligned}
\sum_{\chi}^{*} \Lambda_{22}(\sigma) \leq & \sum_{\chi} \int_{-T}^{T}\left|f_{2}(\sigma+i t)\right|^{2} d t \\
= & 2 \phi(q) \kappa^{2(2 \sigma-k)} \sum_{\substack{n<2 T / \kappa \\
(n, q)=1 \\
n \equiv m(q)}} \sum_{\substack{m<2 T / \kappa \\
(m, q)=1}} \frac{\bar{a}(n) a(m)}{(n m)^{k-\sigma}} \\
& \times \int_{0}^{T} \varphi_{0}\left(\frac{\kappa n}{t}\right) \varphi_{0}\left(\frac{\kappa m}{t}\right)\left|\frac{\Gamma(k-s)}{\Gamma(s)}\right|^{2} \cos \left(t \log \frac{n}{m}\right) d t .
\end{aligned}
$$

Note that the interval $[0, T]$ of integration can be replaced by an interval $[(\kappa / 2) \max (n, m), T]$, because $\varphi_{0}(\kappa n / t) \varphi_{0}(\kappa m / t)=0$ for $0 \leq t \leq$ $(\kappa / 2) \max (n, m)$. By Stirling's formula, we have

$$
\left|\frac{\Gamma(k-s)}{\Gamma(s)}\right|^{2}=|t|^{2(k-2 \sigma)}\left(1+O\left(\frac{1}{t^{2}}\right)\right)
$$


for $0<\sigma<k$ and $|t| \geq C_{1}$, where $C_{1}$ is some positive constant. In case $n$ and $m$ satisfy $C_{1} \leq(\kappa / 2) \max (n, m)$, we have

$$
\begin{aligned}
& \int_{(\kappa / 2) \max (n, m)}^{T} \varphi_{0}\left(\frac{\kappa n}{t}\right) \varphi_{0}\left(\frac{\kappa m}{t}\right)\left|\frac{\Gamma(k-s)}{\Gamma(s)}\right|^{2} \cos \left(t \log \frac{n}{m}\right) d t \\
& =\int_{(\kappa / 2) \max (n, m)}^{T} \varphi_{0}\left(\frac{\kappa n}{t}\right) \varphi_{0}\left(\frac{\kappa m}{t}\right) t^{2(k-2 \sigma)} \cos \left(t \log \frac{n}{m}\right) d t+O(1)
\end{aligned}
$$

uniformly in $\sigma$ and $q$ for $k / 2-1 / \log (q T) \leq \sigma \leq k / 2+1 / \log (q T)$. The same result also holds in case $C_{1}>(\kappa / 2) \max (n, m)$, because in this case

$$
\int_{(\kappa / 2) \max (n, m)}^{C_{1}} \varphi_{0}\left(\frac{\kappa n}{t}\right) \varphi_{0}\left(\frac{\kappa m}{t}\right)\left|\frac{\Gamma(k-s)}{\Gamma(s)}\right|^{2} \cos \left(t \log \frac{n}{m}\right) d t=O(1)
$$

and

$$
\int_{(\kappa / 2) \max (n, m)}^{C_{1}} \varphi_{0}\left(\frac{\kappa n}{t}\right) \varphi_{0}\left(\frac{\kappa m}{t}\right) t^{2(k-2 \sigma)} \cos \left(t \log \frac{n}{m}\right) d t=O(1) .
$$

Let us denote

$$
\sum_{1}=\sum_{\substack{n<2 T / \kappa \\(n, q)=1 \\ n \equiv m(q)}} \sum_{\substack{m<2 T / \kappa \\(m, q)=1}}
$$

and $\sum_{0}$ is as before. From the above result, it follows that

$$
\begin{aligned}
\sum_{1} \frac{\bar{a}(n) a(m)}{(n m)^{k-\sigma}} & \\
& \times \int_{(\kappa / 2) \max (n, m)}^{T} \varphi_{0}\left(\frac{\kappa n}{t}\right) \varphi_{0}\left(\frac{\kappa m}{t}\right)\left|\frac{\Gamma(k-s)}{\Gamma(s)}\right|^{2} \cos \left(t \log \frac{n}{m}\right) d t \\
= & \sum_{1} \frac{\bar{a}(n) a(m)}{(n m)^{k-\sigma}} \\
& \times \int_{(\kappa / 2) \max (n, m)}^{T} \varphi_{0}\left(\frac{\kappa n}{t}\right) \varphi_{0}\left(\frac{\kappa m}{t}\right) t^{2(k-2 \sigma)} \cos \left(t \log \frac{n}{m}\right) d t \\
& +O\left(\sum_{1} \frac{|a(n) a(m)|}{(n m)^{k-\sigma}}\right) \\
= & \sum_{\substack{n<T /(2 \kappa) \\
(n, q)=1}} \frac{|a(n)|^{2}}{n^{2(k-\sigma)}} \int_{0}^{T} \varphi_{0}\left(\frac{\kappa n}{t}\right)^{2} t^{2(k-2 \sigma)} d t
\end{aligned}
$$




$$
\begin{aligned}
& +\sum_{\substack{T /(2 \kappa) \leq n<2 T / \kappa \\
(n, q)=1}} \frac{|a(n)|^{2}}{n^{2(k-\sigma)}} \int_{0}^{T} \varphi_{0}\left(\frac{\kappa n}{t}\right)^{2} t^{2(k-2 \sigma)} d t \\
& +\sum_{0} \frac{\bar{a}(n) a(m)}{(n m)^{k-\sigma}} \int_{0}^{T} \varphi_{0}\left(\frac{\kappa n}{t}\right) \varphi_{0}\left(\frac{\kappa m}{t}\right) t^{2(k-2 \sigma)} \cos \left(t \log \frac{n}{m}\right) d t \\
& +O\left(\sum_{1} \frac{|a(n) a(m)|}{(n m)^{k-\sigma}}\right) .
\end{aligned}
$$

Since $-4 / \log (q T) \leq-2(k-2 \sigma) \leq 4 / \log (q T)$, by using Lemma 4 , we see that the right-hand side of the above is

$$
\begin{aligned}
& \ll T \sum_{n<T /(2 \kappa)} \frac{|a(n)|^{2}}{n^{2(k-\sigma)}}+\frac{1}{q} \sum_{n<2 T / \kappa} \frac{|a(n)|^{2}}{n^{2(k-\sigma)-1}}+\sum_{0} \frac{|a(n) a(m)|}{(n m)^{k-\sigma}\left|\log \frac{n}{m}\right|} \\
& +q \sum_{0} \frac{|a(n) a(m)|}{(n m)^{k-\sigma} \max (n, m)\left(\log \frac{n}{m}\right)^{2}}+\sum_{1} \frac{|a(n) a(m)|}{(n m)^{k-\sigma}}
\end{aligned}
$$

uniformly in $\sigma$ and $q$ for $k / 2-1 / \log (q T) \leq \sigma \leq k / 2+1 / \log (q T)$. By (15), (16), and the estimate

$$
\sum_{1} \frac{|a(n) a(m)|}{(n m)^{k-\sigma}} \leq \sum_{n<2 T / \kappa} \frac{|a(n)|^{2}}{n^{2(k-\sigma)}} \sum_{\substack{m<2 T / \kappa \\ m \equiv n(q)}} 1 \ll T \sum_{n<2 T / \kappa} \frac{|a(n)|^{2}}{n^{2(k-\sigma)}},
$$

we have

$$
\sum_{\chi}^{*} \Lambda_{22}(\sigma) \ll \phi(q)\left(T \sum_{n<2 T / \kappa} \frac{|a(n)|^{2}}{n^{2(k-\sigma)}}+\frac{\log T}{q} \sum_{n<2 T / \kappa} \frac{|a(n)|^{2}}{n^{2(k-\sigma)-1}}\right),
$$

hence, by (6), we obtain

$$
\sum_{\chi}^{*} \Lambda_{22}(\sigma) \ll \phi(q) T \log (q T), \quad T \rightarrow \infty,
$$

uniformly in $\sigma$ and $q$ for $k / 2-1 / \log (q T) \leq \sigma \leq k / 2+1 / \log (q T)$.

Combining (13), (17), (18), and the assumption $q \ll T$, we obtain the assertion of Theorem 2.

COROllary 1. Under the same notation as in Theorem 2, we have

$$
\sum_{\chi}^{*} \int_{-T}^{T}\left|L_{f}^{\prime}(k / 2+i t, \chi)\right|^{2} d t \ll \phi(q) T(\log (q T))^{3}, \quad T \rightarrow \infty,
$$

uniformly in $q$. 
Proof. Put $r=(\log (q T))^{-1}$. Since

$$
\left|L_{f}^{\prime}(k / 2+i t, \chi)\right|^{2} \ll r^{-3} \int_{|z-k / 2-i t|=r}\left|L_{f}(z, \chi)\right|^{2}|d z|,
$$

we have

$$
\begin{array}{rl}
\sum_{\chi}^{*} \int_{-T}^{T}\left|L_{f}^{\prime}(k / 2+i t, \chi)\right|^{2} & d t \\
& \ll r^{-3} \sum_{\chi}^{*} \int_{-T|z-k / 2-i t|=r}^{T} \int_{f}\left|L_{f}(z, \chi)\right|^{2}|d z| d t .
\end{array}
$$

From Theorem 2, it follows that

$$
\begin{aligned}
& \sum_{\chi}^{*} \int_{-T}^{T} \int_{|z-k / 2-i t|=r}\left|L_{f}(z, \chi)\right|^{2}|d z| d t \\
& \leq 2 \int_{k / 2-r}^{k / 2+r} \sum_{\chi}^{*} \int_{-T-1}^{T+1}\left|L_{f}(\sigma+i t, \chi)\right|^{2} d t\left(1-\left(\frac{\sigma-k / 2}{r}\right)^{2}\right)^{-1 / 2} d \sigma \\
& \leq 2\left\{\int_{k / 2-r}^{k / 2+r}\left(\sum_{\chi}^{*} \int_{-T-1}^{T+1}\left|L_{f}(\sigma+i t, \chi)\right|^{2} d t\right)^{3} d \sigma\right\}^{1 / 3} \\
& \quad \times\left\{\int_{k / 2-r}^{k / 2+r}\left(1-\left(\frac{\sigma-k / 2}{r}\right)^{2}\right)^{-3 / 4} d \sigma\right\}^{2 / 3} \\
& \ll r \phi(q) T \log (q T) \ll \phi(q) T .
\end{aligned}
$$

This proves the corollary.

Corollary 2. Let $\chi$ be a Dirichlet character $\bmod q$, and $\chi^{*}$ the primitive character inducing $\chi$. Let $\delta$ be a positive real number such that $\delta \ll T$, and $\mathcal{T}_{\chi^{*}}$ a finite subset of $[-T, T]$ with $\left|t-t^{\prime}\right| \geq \delta$ for any distinct $t$ and $t^{\prime}$ in $\mathcal{T}_{\chi^{*}}$. If $q \ll T$, then

$$
\sum_{\chi} \sum_{t \in \mathcal{T}_{\chi^{*}}}\left|L_{f}\left(k / 2+i t, \chi^{*}\right)\right|^{2} \ll\left(\frac{1}{\delta}+\log (q T)\right) q T \log (q T), \quad T \rightarrow \infty,
$$

uniformly in $q$.

Corollary 2 can be derived from Theorem 2 and Corollary 1 by the same argument as the proof of Corollary 10.4 of [6].

4. Proof of Theorem 1. Our argument is a modification of the proof of the zero density estimates of Dirichlet $L$-functions in [6], so we give only a sketch. 
Let $L_{f}(s)=\sum_{n=1}^{\infty} a(n) n^{-s}$ for $\sigma>(k+1) / 2$. We define $\mu_{f}(n)$ by

$$
\frac{1}{L_{f}(s)}=\sum_{n=1}^{\infty} \frac{\mu_{f}(n)}{n^{s}}
$$

for $\sigma>(k+1) / 2$. By the Euler product expansion of $L_{f}(s)$ and the estimate $|a(n)| \leq n^{(k-1) / 2} d(n)$ (see [2]), where $d(n)$ is the divisor function, it is easy to see that the following properties hold:

$$
\begin{gathered}
\left|\mu_{f}(n)\right| \leq n^{(k-1) / 2} d(n) \\
\sum_{\substack{d \mid n \\
d>0}} \mu_{f}(d) a\left(\frac{n}{d}\right)= \begin{cases}1 & \text { if } n=1 \\
0 & \text { otherwise }\end{cases}
\end{gathered}
$$

Since $L_{f}(s, \chi)$ and $L_{f}\left(s, \chi^{*}\right)$ have the same zeros for $\sigma \geq k / 2$, it is enough to consider $N_{f}\left(\sigma_{0}, T, \chi^{*}\right)$ instead of $N_{f}\left(\sigma_{0}, T, \chi\right)$. Let $A_{1}$ be a positive real number, and let $X$ and $Y$ be parameters satisfying $2 \leq X \leq Y \leq(q T)^{A_{1}}$. We define

$$
M\left(s, \chi^{*}\right)=\sum_{n \leq X} \frac{\mu_{f}(n) \chi^{*}(n)}{n^{s}} .
$$

Then it follows that, for $\sigma>(k+1) / 2$,

$$
L_{f}\left(s, \chi^{*}\right) M\left(s, \chi^{*}\right)=\sum_{n=1}^{\infty} \frac{h(n) \chi^{*}(n)}{n^{s}},
$$

where $h(n)=\sum_{d \mid n, 0<d \leq X} \mu_{f}(d) a(n / d)$ has the following properties: $h(1)=1$, $h(n)=0$ for $2 \leq n \leq X$, and $|h(n)| \leq n^{(k-1) / 2} d(n)^{3}$ for $n>X$. By using the Mellin integral formula, we have

$$
\begin{aligned}
e^{-1 / Y}+ & \sum_{n>X} h(n) \chi^{*}(n) n^{-s} e^{-n / Y} \\
& =\frac{1}{2 \pi i} \int_{(k+1) / 2+1-i \infty}^{(k+1) / 2+1+i \infty} L_{f}\left(s+w, \chi^{*}\right) M\left(s+w, \chi^{*}\right) Y^{w} \Gamma(w) d w
\end{aligned}
$$

for $\sigma>-1$. Let $\varrho=\beta+i \gamma$ be a zero of $L_{f}\left(s, \chi^{*}\right)$ such that $\sigma_{0} \leq \beta \leq$ $(k+1) / 2$ and $-T \leq \gamma \leq T$, and take $s=\varrho$ in the equation above. Since $L_{f}\left(\varrho+w, \chi^{*}\right) M\left(\varrho+w, \chi^{*}\right) Y^{w} \Gamma(w)$ is holomorphic for $-1 / 2 \leq \Re w$, the path of integration in the above can be moved to the line $\Re w=k / 2-\beta$. Therefore, if $Y$ is large, every $\varrho$ counted by $N_{f}\left(\sigma_{0}, T, \chi^{*}\right)$ has at least one of the following properties:

$$
\left|\sum_{X<n \leq Y^{2}} h(n) \chi^{*}(n) n^{-\varrho} e^{-n / Y}\right| \geq \frac{1}{5},
$$




$$
\left|\int_{k / 2-\beta-i z}^{k / 2-\beta+i z} L_{f}\left(\varrho+w, \chi^{*}\right) M\left(\varrho+w, \chi^{*}\right) Y^{w} \Gamma(w) d w\right| \geq \frac{2 \pi}{5},
$$

where $z=A_{2} \log (q T)$ for a large absolute constant $A_{2}$. Let $\mathcal{R}\left(\chi^{*}\right)$ be a set of $\varrho$ 's which are well-spaced, that is, $3 z \leq\left|\gamma-\gamma^{\prime}\right|$ for any distinct $\varrho=\beta+i \gamma$ and $\varrho^{\prime}=\beta^{\prime}+i \gamma^{\prime}$. We denote by $R\left(\chi^{*}\right)$ the number of elements of $\mathcal{R}\left(\chi^{*}\right)$. From (2) and the definition of $\mathcal{R}\left(\chi^{*}\right)$, it follows that

$$
N_{f}\left(\sigma_{0}, T, \chi^{*}\right) \ll R\left(\chi^{*}\right)(\log (q T))^{2},
$$

hence

$$
\sum_{\chi} N_{f}\left(\sigma_{0}, T, \chi\right)=\sum_{\chi} N_{f}\left(\sigma_{0}, T, \chi^{*}\right) \ll R(\log (q T))^{2},
$$

where $R=\sum_{\chi} R\left(\chi^{*}\right)$. The sets $\mathcal{R}_{1}\left(\chi^{*}\right)$ and $\mathcal{R}_{2}\left(\chi^{*}\right)$ are defined to be the subsets of $\mathcal{R}\left(\chi^{*}\right)$ such that every element of $\mathcal{R}_{1}\left(\chi^{*}\right)$ satisfies the condition (a), and every element of $\mathcal{R}_{2}\left(\chi^{*}\right)$ satisfies the condition (b). Denote by $R_{j}\left(\chi^{*}\right)$ the number of elements of $\mathcal{R}_{j}\left(\chi^{*}\right), j=1,2$. Put

$$
\mathcal{R}_{j}=\bigcup_{\chi} \mathcal{R}_{j}\left(\chi^{*}\right) \quad \text { and } \quad R_{j}=\sum_{\chi} R_{j}\left(\chi^{*}\right), \quad j=1,2,
$$

and we shall estimate $R_{1}$ and $R_{2}$.

First, we estimate $R_{1}$. For every $\varrho$ in $\mathcal{R}_{1}$,

$$
\max _{1 \leq l \leq l_{0}+1}\left\{\left|\sum_{\substack{X<n \leq 2^{l} X \\ 2^{l-1} \leq Y^{2}}} h(n) \chi^{*}(n) n^{-\varrho} e^{-n / Y}\right|\right\} \geq \frac{1}{15 \log Y}
$$

for large $Y$, where $l_{0}=\left[(\log 2)^{-1} \log \left(X^{-1} Y^{2}\right)\right]$. Hence, there exists $U$ such that $X<U \leq Y^{2}$ and the inequality

$$
\left|\sum_{\substack{U<n \leq 2 U \\ n \leq Y^{2}}} h(n) \chi^{*}(n) n^{-\varrho} e^{-n / Y}\right| \geq \frac{1}{15 \log Y}
$$

holds for more than $R_{1} /(4 \log Y)$ zeros of $\mathcal{R}_{1}$. Therefore, by Theorem 7.6 of $[6]$,

$$
\begin{aligned}
R_{1} & \ll(\log Y)^{3} \sum_{\chi} \sum_{\varrho \in \mathcal{R}_{1}\left(\chi^{*}\right)}\left|\sum_{\substack{U<n \leq 2 U \\
n \leq Y^{2}}} h(n) \chi^{*}(n) n^{-\varrho} e^{-n / Y}\right|^{2} \\
& \ll\left(q T X^{k-2 \sigma_{0}}+Y^{k+1-2 \sigma_{0}}\right)(\log (q T))^{67}
\end{aligned}
$$


Second, we estimate $R_{2}$. For every $\varrho$ in $\mathcal{R}_{2}$,

$$
\begin{aligned}
\int_{-z}^{z} \mid L_{f}\left(k / 2+i(\gamma+v), \chi^{*}\right) M(k / 2 & \left.+i(\gamma+v), \chi^{*}\right) \mid \\
& \times Y^{k / 2-\beta}|\Gamma(k / 2-\beta+i v)| d v \geq \frac{2 \pi}{5} .
\end{aligned}
$$

Let $t_{\varrho}=\gamma+v$ be a value for which $\mid L_{f}\left(k / 2+i(\gamma+v), \chi^{*}\right) M(k / 2+i(\gamma+$ $\left.v), \chi^{*}\right) \mid$ is maximal. Since

$$
\int_{-z}^{z}|\Gamma(k / 2-\beta+i v)| d v \ll \int_{-1}^{1} \frac{1}{\beta-k / 2} d v \ll \log (q T),
$$

we have

$$
\left|L_{f}\left(k / 2+i t_{\varrho}, \chi^{*}\right) M\left(k / 2+i t_{\varrho}, \chi^{*}\right)\right| \gg Y^{\sigma_{0}-k / 2}(\log (q T))^{-1} .
$$

Hence,

$$
\begin{aligned}
Y^{\sigma_{0}-k / 2}(\log (q T))^{-1} R_{2} \ll & \sum_{\chi} \sum_{\varrho \in \mathcal{R}_{2}\left(\chi^{*}\right)}\left|L_{f}\left(k / 2+i t_{\varrho}, \chi^{*}\right) M\left(k / 2+i t_{\varrho}, \chi^{*}\right)\right| \\
\leq & \left(\sum_{\chi} \sum_{\varrho \in \mathcal{R}_{2}\left(\chi^{*}\right)}\left|L_{f}\left(k / 2+i t_{\varrho}, \chi^{*}\right)\right|^{2}\right)^{1 / 2} \\
& \times\left(\sum_{\chi} \sum_{\varrho \in \mathcal{R}_{2}\left(\chi^{*}\right)}\left|M\left(k / 2+i t_{\varrho}, \chi^{*}\right)\right|^{2}\right)^{1 / 2} .
\end{aligned}
$$

Since $\left|t_{\varrho}-t_{\varrho^{\prime}}\right| \geq z$, we can use Corollary 2 under the assumption $q \ll T$ :

$$
\sum_{\chi} \sum_{\varrho \in \mathcal{R}_{2}\left(\chi^{*}\right)}\left|L_{f}\left(k / 2+i t_{\varrho}, \chi^{*}\right)\right|^{2} \ll q T(\log (q T))^{2} .
$$

From Theorem 7.6 of [6], if $X \leq q T$, then

$$
\sum_{\chi} \sum_{\varrho \in \mathcal{R}_{2}\left(\chi^{*}\right)}\left|M\left(k / 2+i t_{\varrho}, \chi^{*}\right)\right|^{2} \ll q T(\log (q T))^{6} .
$$

Therefore, if $q \ll T$ and $X \leq q T$, we obtain

$$
R_{2} \ll Y^{k / 2-\sigma_{0}} q T(\log (q T))^{5} .
$$

Substituting (20) and (21) into (19) gives

$$
\sum_{\chi} N_{f}\left(\sigma_{0}, T, \chi\right) \ll\left(q T X^{k-2 \sigma_{0}}+Y^{k+1-2 \sigma_{0}}+q T Y^{k / 2-\sigma_{0}}\right)(\log (q T))^{69},
$$

and putting $X=q T, Y=(q T)^{1 /\left(k / 2+1-\sigma_{0}\right)}$, we now obtain (3) uniformly in $\sigma_{0}$ and $q$ for $k / 2+1 / \log (q T) \leq \sigma_{0} \leq(k+1) / 2$. 
Finally, the estimate (4) can be derived by a different treatment of $R_{1}$ and $R_{2}$. This is almost identical to the proof of Theorem 12.1 of [6], so we omit the details.

\section{References}

[1] H. Daven port, Multiplicative Number Theory, 2nd ed., Grad. Texts in Math. 74, Springer, Berlin, 1980.

[2] P. Deligne, La conjecture de Weil, Inst. Hautes Études Sci. Publ. Math. 43 (1974), 273-307.

[3] A. Good, Approximative Funktionalgleichungen und Mittelwertsätze für Dirichletreihen, die Spitzenformen assoziiert sind, Comment. Math. Helv. 50 (1975), 327-361.

[4] A. Ivić, On zeta-functions associated with Fourier coefficients of cusp forms, in: Proceedings of the Amalfi Conference on Analytic Number Theory, E. Bombieri et al. (eds.), Università di Salerno, 1992, 231-246.

[5] T. Miyake, Modular Forms, Springer, Berlin, 1989.

[6] H. L. Montgomery, Topics in Multiplicative Number Theory, Lecture Notes in Math. 227, Springer, Berlin, 1971.

[7] R. A. Rankin, Contributions to the theory of Ramanujan's function $\tau(n)$ and similar arithmetical functions II, Proc. Cambridge Philos. Soc. 35 (1939), 357-372.

Graduate School of Mathematics

Nagoya University

Chikusa-ku, Nagoya 464-01, Japan

E-mail: m95011@math.nagoya-u.ac.jp

Received on 5.5.1997

and in revised form on 13.2.1998 